\title{
Steps along the road to electrochemical devices for early cancer diagnosis
}

\author{
"Accurate measurement of panels of protein biomarkers has the potential for early detection and \\ directing individualized cancer therapy."
}

\section{The challenge of early cancer detection using biomarkers}

Proteins overexpressed by cancer cells and secreted into the bloodstream can serve as biomarkers to detect cancer [1,2]. Accurate measurement of panels of protein biomarkers has the potential for early detection and directing individualized cancer therapy [3]. Reliable prediction will ultimately require devices that measure a small number (e.g., 4-10) of biomarker proteins for each cancer. Devices need to be accurate, sensitive, cheap and easy to use at point-of-care in order to facilitate rapid diagnosis, minimize sample decomposition and decrease patient anxiety.

Realization of critical issues can help us understand how to achieve these goals. First, measurement of panels of biomarkers in serum for a given cancer provides much better prediction success than measurement of a single biomarker [1-5]. Second, many protein biomarkers are indicative of more than one disease, such as IL-6, which is overexpressed in oral, prostate, lung, multiple myeloma and renal cancers [6]. Third, there are widely different concentrations of biomarker proteins in serum, ranging from under $1 \mathrm{pg} / \mathrm{ml}$ to hundreds of $\mathrm{ng} / \mathrm{ml}[6,7]$. Finally, strategies are needed to prevent the thousands of other proteins in serum from interfering with biomarker assays [1]. Thus, developing pointof-care bioanalytical devices for early cancer diagnosis represents a considerable challenge.

How do we presently stand in terms of methodology for multiple protein detection? Historically, ELISAs served as workhorse methods with detection limits (DLs) approaching $1 \mathrm{pg} / \mathrm{ml}[8]$. However, ELISA, along with powerful LC-MS proteomics methods, are not directly applicable for point-of-care, owing to equipment cost, assay time and the difficulty of multiplexing.

ELISA-like approaches have been successfully adapted to immunoarrays, most often using sandwich immunoassays (FIGURE I). Microarrays relying on optical or electrical detection have considerable promise for achieving point-of-care measurements [8-10]. SPR and nanowire transistor arrays offer exciting future possibilities, but are, as yet, far from point-of-care applications. This editorial addresses the promise of electrochemical protein microarrays, which can provide high selectivity and sensitivity, ease of use and low cost, and are also amenable to automation.

\section{High-sensitivity electrochemical protein measurements}

In electrochemical immunosensors, primary antibodies $\left(A b_{1}\right)$ or aptamers are attached to an electrode surface to capture analyte proteins from the sample. After washing with a protein/detergent solution to block nonspecific binding (NSB), a labeled secondary antibody $\left(A b_{2}\right)$ is added to bind to analyte proteins on the sensor or array (FIGURE I). High sensitivity has been a major focus of recent research on these immunosensors and multiple-label methods have enabled exquisite DL at or below $\mathrm{pg} / \mathrm{ml}$ (fM) levels. Among the most successful of these employ dissolvable inorganic nanoparticles or nanoparticles with multiple enzyme labels [11].

Metal or metal-sulfide nanoparticle labels are subsequently dissolved to release thousands of metal ions that are detected by electrochemical stripping analysis. Alternatively, nanoparticles such as carbon nanotubes or magnetic iron oxide can be bioconjugated with thousands of enzymes to detect electroactive products or the enzymes themselves [11,12]. Multiple enzyme methods employ a single catalytic detection step as opposed to the dissolution, preconcentration and stripping steps in the inorganic nanoparticle approach. Finally, label-free electrochemical impedance spectroscopy and potential step capacitance hold the promise of simplicity, although achieving ultrahigh sensitivity is in early stages [13]. A problem with many of the

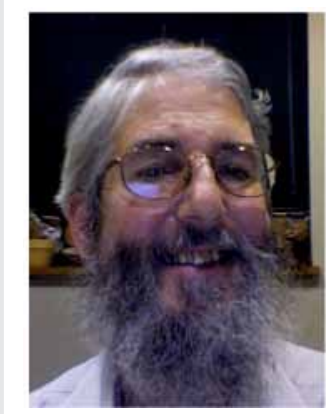

James F Rusling ${ }^{1,2,3}$

'Department of Chemistry, University of Connecticut,

55 North Eagleville Road, Storrs, Connecticut 06269, USA

${ }^{2}$ Institute of Materials Science, University of Connecticut, Storrs, USA

${ }^{3}$ Department of Cell Biology, University of Connecticut Health Center, Farmington, CT, USA Tel.: + I 8604864909

E-mail: james.rusling@uconn.edu 


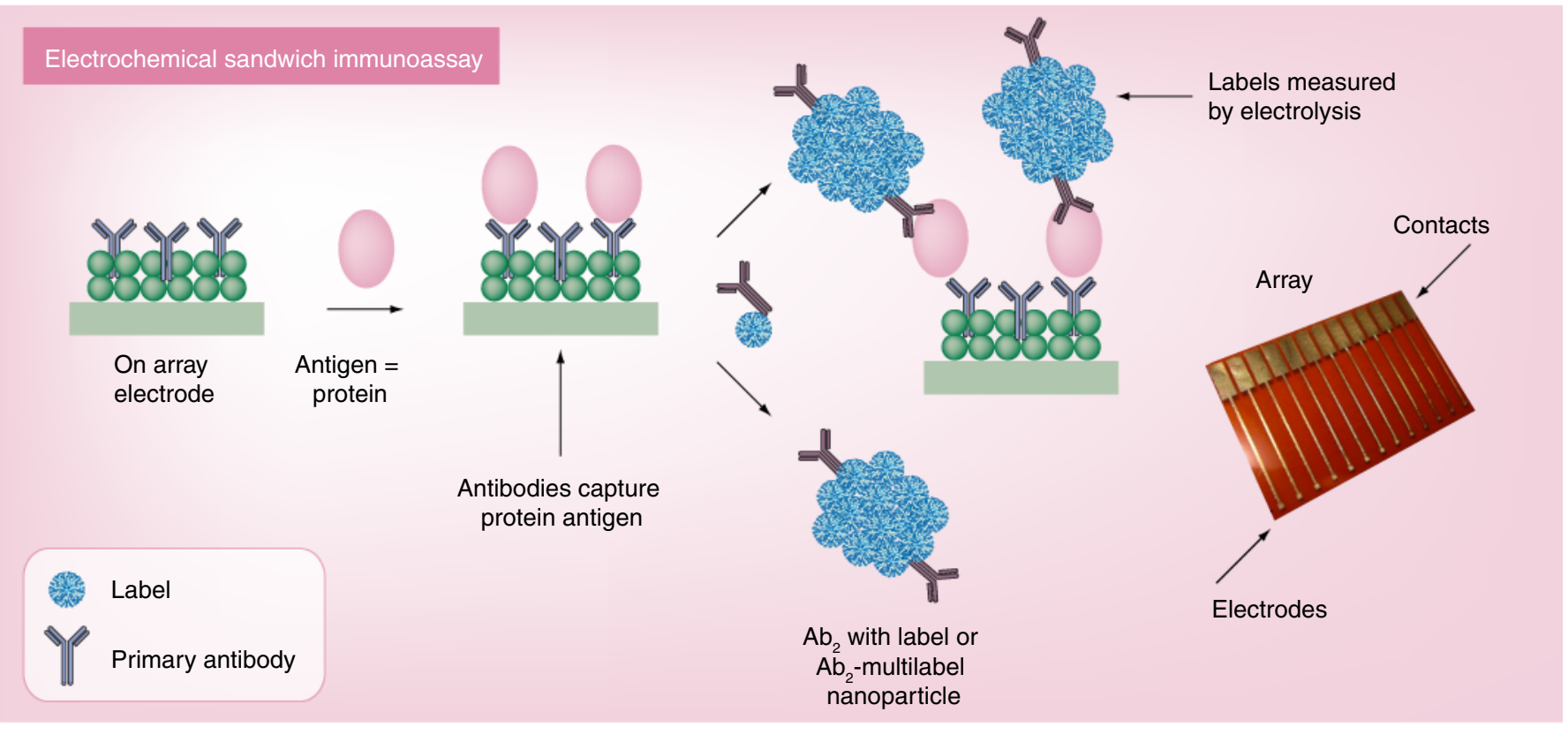

Figure 1. Representative illustration of high-sensitivity electrochemical immunoarrays. Primary antibodies $\left(\mathrm{Ab}_{1}\right)$ are attached to a nanostructured electrode surface to capture analyte proteins from the sample. After washing to block nonspecific binding, a labeled $A b_{2}$ or, for higher sensitivity, a multilabel nanoparticle- $A b_{2}$ bioconjugate is added to bind to analyte proteins on the array. Labels can be enzymes, redox probes or inorganic materials that are detected by an appropriate electroanalytical method (see text). $\mathrm{Ab}_{2}$ : Secondary antibody.

systems already mentioned is that very few have been optimized for biomedical samples, so performance in practical analyses is uncertain.

Electrochemiluminescence (ECL) methods have achieved high sensitivity protein detection; tris(2,2'-bipyridyl)ruthenium(II) (RuBPY) produces ECL light when its $\mathrm{Ru}^{\mathrm{III}}$ form reacts with a reductant [14]. Magnetic bead assays employing RuBPY-labeled secondary antibodies $\left(\mathrm{Ab}_{2}\right)$ are marketed by several companies. Automated flow systems and kits are available for up to ten selected proteins in serum and other biological media. However, current automated technology is expensive, requires considerable maintenance and is more suited for research and hospital laboratories than for point-of-care situations.

\section{Progress toward point-of-care electrochemical immunoarrays}

Translating ultrasensitive protein detection into multiprotein formats has lagged well behind single protein immunosensors. One possibility is a barcode-like approach based on labeling $\mathrm{Ab}_{2}$ with inorganic nanoparticles having distinct electrochemical potentials that are dissolved and detected by stripping methods $[4,11]$. Alternatively, microelectronic arrays of multiple electrodes attached to distinct antibodies for a range of proteins can be used.
An example of the inorganic nanoparticle method employed $\mathrm{CdS}, \mathrm{ZnS}, \mathrm{CuS}$ and $\mathrm{PbS}$ nanoparticles attached to four different $\mathrm{Ab}_{2}$ to detect four different proteins [11]. Individual metal ions each code for a different protein and were measured by stripping voltammetry after dissolution of the particle. A variation employs multiple-metal spheres or rods as labels. These labels give a series of stripping peaks with potentials and relative intensities characteristic of each analyte protein.

Illustrating microelectronic arrays, eightelectrode iridium oxide arrays with capture antibodies attached were used to develop enzyme-labeled sandwich immunoassays for simultaneous detection of seven cancer biomarkers with DLs of approximately $2 \mathrm{ng} / \mathrm{ml}$ [15] . The method showed good correlation to ELISA for a standard serum sample. CombiMatrix chips with more than 1000 electrodes/cm have also been used for immunoassays that included protein analytes [16]. Oligonucleotides were synthesized on $100-\mu \mathrm{m}$ electrodes, and complementary oligonucleotide strands on $\mathrm{Ab}_{2}$ bound them onto specific electrodes. This method was used to simultaneously determine human $\alpha 1$ acid glycoprotein, ricin, M13 phage, Bacillus globigii spores and fluorescein with a $5-\mathrm{pg} / \mathrm{ml}$ DL for APG. These chips have the potential for 
massive parallel detection of thousands of proteins, but are currently limited by the necessity of attaching oligonucleotide strands to a large number of antibodies.

Wong et al. used arrays to detect the oral cancer biomarkers IL-8 protein and mRNA in saliva. Arrays featured a DNA dendrimer/conducting polymer film to lower NSB and provide binding sites for capture antibodies [17]. IL-8, IL- $1 \mathrm{~b}$ and IL- 8 mRNA were measured in buffer with DLs of $100-200 \mathrm{fg} / \mathrm{ml}$ for the proteins and $10 \mathrm{aM}$ for IL-8 mRNA. For saliva, considerably higher DLs for IL-8mRNA ( 4 fM) and IL-8 $(7.4 \mathrm{pg} / \mathrm{ml})$ were found [18]. Analysis of oral cancer saliva samples and controls gave approximately $90 \%$ sensitivity and specificity for both analytes. These studies illustrate the importance of testing real biomedical samples, as they reveal considerable degradation of $\mathrm{DL}$ when switching from buffer to saliva.

Our research team used a four-unit electrochemical immunoarray equipped with nanostructured carbon nanotube electrodes to measure four prostate cancer biomarkers in cancer patient serum [19]. Sensitivity was tailored to analyte requirements by combining single- and multiple-labeled strategies. $\mathrm{Ab}_{2}$ with 14-16 horseradish peroxidase (HRP) labels were used to obtain necessary sensitivity for protein biomarkers platelet factor-4 (PF-4) $(\mathrm{DL} \sim 1 \mathrm{ng} / \mathrm{ml})$ and IL-6 (DL $\sim 30 \mathrm{pg} / \mathrm{ml})$. Singly labeled $\mathrm{Ab}_{2}-\mathrm{HRP}$ was suitable for prostate-specific antigen and prostate-specific membrane antigen. Determinations of these four proteins in the serum of prostate cancer patients and controls gave excellent correlations to ELISA [19].

\section{Summary \& future perspective}

Clearly, research efforts in electrochemical protein detection have achieved the necessary ultrahigh sensitivity. Electrochemical sandwich immunoassays provide high sensitivity and selectivity with the potential for multiplexing. Unfortunately, except for ECL bead assays, reports in which highly sensitive electrochemical immunoarrays have been validated using real patient samples are few [12,18-20]. However, commercial ECL technology does not meet point-of-care criteria, as discussed above. Further, electrochemical stripping detection presents limitations in complexity and analysis time. While label-free impedance/capacitance methods are attractively simple, unsolved problems with NSB remain.
From this viewpoint, sandwich immunoassays on microfabricated multi-electrode chips have the advantages of low cost, high sensitivity, accuracy and simplicity. Multi- and singlelabel $\mathrm{Ab}_{2}$ systems can be tailored to different panels of biomarker analytes to accommodate ultralow- and high-concentration proteins in the sample. It might also be possible to fabricate simple ECL arrays with the required sensitivity and accuracy.

To reach widespread point-of-care use, any device will need to be automated as much as possible. Integration into simple microfluidic systems might serve well for that purpose. Furthermore, all devices intended for clinical use must be fully validated for predictive sensitivity and selectivity by using patients' samples.

With multiple alternatives for ultrahigh sensitivity electrochemical immunosensors and the beginnings of translation to multiprotein arrays, the first few big steps along the road to electrochemical arrays for cancer detection and monitoring have been taken; however, significant challenges lie ahead. They involve reliable microarray fabrication and integration into automated systems, optimization for serum and other real samples and increased speed of analysis. We have not stressed the latter point, but point-of-care testing should be fast enough to relay results quickly. Assay speed also opens up other doors, such as aiding in surgical decisions.

In summary, there are significant challenges to meet before point-of-care electrochemical arrays for cancer diagnosis become a reality. A few major challenges have already been met and progress is good and the future is bright for producing arrays within the next decade that can be used for early cancer detection.

\section{Acknowledgements}

The author is grateful for contributions of all co-workers in the protein immunoarray development project as named in joint publications.

Financial \& competing interests disclosure The author's work in this area is supported by PHS grant ES013557 from NIEHS/NIH. The author has no other relevant affiliations or financial involvement with any organization or entity with a financial interest in or financial conflict with the subject matter or materials discussed in the manuscript apart from those disclosed.

No writing assistance was utilized in the production of this manuscript. 


\section{Bibliography}

1 Kulasingam V, Diamandis EP. Strategies for discovering novel cancer biomarkers through utilization of emerging technologies. Nat. Clin. Pract. Oncol. 5, 588-599 (2008).

2 Hanash SM, Pitteri SJ, Faca VM. Mining the plasma proteome for cancer biomarkers. Nature 452, 571-579 (2008).

3 Ludwig JA, Weinstein JN. Biomarkers in cancer staging, prognosis and treatment selection. Nat. Rev. Cancer 5, 845-856 (2005).

4 Tothill IE. Biosensors for cancer markers diagnosis. Semin. Cell Dev. Biol. 20, 55-62 (2009).

5 Wang J, Electrochemical biosensors: towards point-of-care cancer diagnostics. Biosens. Bioelectron. 21, 1887-1892 (2006).

6 Riedel F, Zaiss I, Herzog D, Götte K, Naim R, Hörman K. Serum levels of interleukin 6 in patients with primary head and neck squamous cell carcinoma. Anticancer Res. 25, 2761-2766 (2005).

7 Lilja H, Ulmert D, Vickers AJ. Prostatespecific antigen and prostate cancer: prediction, detection and monitoring. Nat. Rev. Cancer 8, 268-278 (2008).
8 Kingsmore SF. Multiplexed protein measurement: technologies and applications of protein and antibody arrays. Nat. Rev. Drug Discov. 5, 310-320 (2006).

9 Wilson DS, Nock S. Recent developments in protein microarray technology. Angew. Chem. Int. Ed. 42, 494-500 (2003).

10 Lee HJ, Wark AW, Corn RM. Microarray methods for protein biomarker detection. Analyst 133, 975-983 (2008).

11 Wang J. Nanoparticle-based electrochemical bioassays of proteins nanoparticle-based electrochemical bioassays of proteins. Electroanalysis 19, 769-776 (2007).

12 Kim SN, Rusling JF, Papadimitrakopoulos F. Carbon nanotubes in electronic and electrochemical detection of biomolecules. Adv. Mater. 19, 3214-3228 (2007).

13 Tkac J, Davis JJ. Label-free field effect protein sensing. In: Engineering the Bioelectronic Interface. Davis JJ (Ed.). Royal Society of Chemistry, London, UK, 193-224 (2009).

14 Debad JB, Glezer EN, Leland JK, Sigal GB, Wholstadter J. Clinical and biological applications of ECL. In: Electrogenerated Chemiluminescence. Bard AJ (Ed.). Marcel Dekker, NY, USA, 359-396 (2004).
15 Wilson MS, Nie W. Multiplex measurement of seven tumor markers using an electrochemical protein chip. Anal. Chem. 78, 6476-6483 (2006).

16 Dill K, Montgomery DD, Ghindilis AL, Schwarzkopf KR, Ragsdale SR, Oleinikov AV. Immunoassays based on electrochemical detection using microelectrode arrays. Biosens. Bioelectron. 20, 736-742 (2004).

17 Wei F, Liao W, Xu Z, Yang Y, Wong DT, Ho CM. Bio/abiotic interface constructed from nanoscale DNA dendrimer and conducting polymer for ultrasensitive biomolecular diagnosis. Small 5, 1784-1790 (2009).

18 Wei F, Patel P, Liao W et al. Electrochemical sensor for multiplex biomarkers detection. Clin. Cancer Res. 15, 4446-4452 (2009).

19 Chikkaveeriah BV, Bhirde A, Malhotra R, Patel V, Gutkind JS, Rusling JF. Single-wall carbon nanotube forest arrays for immunoelectrochemical measurement of 4 protein biomarkers for prostate cancer. Anal. Chem. 81, 9129-9134 (2009).

20 Mani V, Chikkaveeriah BV, Patel V, Gutkind JS, Rusling JF. Ultrasensitive immunosensor for cancer biomarker proteins using gold nanoparticle film electrodes and multienzymeparticle amplification. ACS Nano 3, 585-594 (2009). 